\title{
A Study of the Initial Stages of Co Deposition on a Silver Electrode in Ammonia Medium Using an Electrochemical Quartz Crystal Microbalance
}

\author{
A. Montes-Rojas and A. L. Donjuan-Medrano \\ Laboratorio de Electroquímica, Centro de Investigación y Estudios de Posgrado, Facultad de Ciencias Químicas, \\ Universidad Autónoma de San Luis Potosí, Avenue Dr. Manuel Nava No. 6, Zona Universitaria, 78210 San Luis Potosi, SLP, Mexico
}

Correspondence should be addressed to A. Montes-Rojas; antonio.montes@uaslp.mx

Received 6 November 2012; Accepted 4 December 2012

Academic Editors: C. M. Muller and W.-S. Zhang

Copyright (C 2013 A. Montes-Rojas and A. L. Donjuan-Medrano. This is an open access article distributed under the Creative Commons Attribution License, which permits unrestricted use, distribution, and reproduction in any medium, provided the original work is properly cited.

\begin{abstract}
The early stages of Co deposition on a silver electrode in ammonia medium were studied using cyclic voltammetry and chronoamperometry coupled with quartz crystal microbalance (EQCM) in ammonia solution. The results obtained by means of EQCM showed that during the initial stages of cobalt deposition a monolayer is formed on the substrate both in the underpotential and overpotential region, and this monolayer is formed at $-600 \mathrm{mV}$ and $-980 \mathrm{mV}$. Once the cobalt deposition process starts, the growth is very fast making the investigation of the initial stages rather difficult. During this process, cobalt atoms transfer their two electrons through free species and not through cobalt hydroxide species adsorbed on the electrode as $\mathrm{CoOH} \mathrm{H}^{+}$or $\mathrm{Co}(\mathrm{OH})_{2}$. In addition, it has been found that at potentials more positive than $-600 \mathrm{mV}$, ammonia adsorption takes place on the substrate surface, and theses species are replaced when the cobalt atoms arrive at potentials more negative than $-600 \mathrm{mV}$.
\end{abstract}

\section{Introduction}

Cobalt is an element that involves a great interest due to its physical and chemical properties and technological applications. Some of its uses are as a catalyst in different reactions of technological interest or as a pigment for strong dark blue color. Also, this element is used in permanent magnet production as well as for preparation of super alloys of great hardness and resistant to high temperatures [1].

One of the properties of this element, when combined with another metal, is the giant magnetoresistance (GMR) whose study has drawn the attention of diverse research teams [2-12]. The importance of this effect lies in that applications of these systems include domains such as informatics, for preparation of reading devices, or technology, for magnetic sensor production. On the basis of these studies it has been possible to show [3] that GMR occurs in materials formed by one magnetic element $(\mathrm{Ni}, \mathrm{Fe}$, or $\mathrm{Co})$ and another nonmagnetic material $(\mathrm{Au}, \mathrm{Ag}, \mathrm{Pd}, \mathrm{Cu}$, etc.). Until now, the commercial preparation of these materials has been preferably performed by metal evaporation techniques [46], in which to avoid undesirable contaminations the whole system must be subject to high-vacuum conditions that generate high production costs.

Because of this, electrodeposition of these materials using a mixed bath is explored with the aim of making their elaboration cheaper. Unlike the techniques of metallic evaporation, electrochemical methods allow us to work in conditions that are similar to those of the environment as well as to reduce the risks of contamination under inert and easily controllable conditions. Additionally, thanks to these methods, the film growth process can be known, and the quantity of deposited material can be controlled by the charge passing through the electrode using electrochemical techniques coupled with quartz microbalance.

Notwithstanding this, some authors [7] have claimed that the magnetic properties sought depend on the method of preparation.

One of the systems that have been studied to find out its magnetic properties is the system formed by cobalt and silver. 
TABLE 1: Extraction works values for cobalt and silver at eV.

\begin{tabular}{lllll}
\hline Metal & $\mathrm{eV}^{15}$ & $\mathrm{eV}^{16}$ & $\mathrm{eV}^{17}$ & $\mathrm{eV}^{18}$ \\
\hline $\mathrm{Ag}$ & 4.78 & 4.71 & 4.58 & 4.71 \\
$\mathrm{Co}$ & 4.70 & 4.25 & 4.18 & 4.16 \\
\hline
\end{tabular}

In the literature, there are several works on the preparation of this system by physical methods [8, 9]; however, only few $[7,10-12]$ have used electrochemical methods to prepare it in a three-dimensional (granular) morphology. One of the most outstanding results, according to the authors [7], is that magnetic properties do not have the same intensity as when obtained by physical methods due to the fact that cobalt inclusions into the silver matrix do not have the same size or homogeneous distribution. These authors state that for a better control of GMR properties of the material, the first stages of its formation have to be known very well.

Since in the preparation of this type of materials using electrochemical technique the growth of one metal takes place on a metal substrate of different nature, and the underpotential deposition process may be considered [13, 14]. According to the bibliography, there are no reports on results that support this idea; however, values obtained from extraction works (electron extraction from the substrate $S$, $\phi_{\mathrm{S}}$, and from the metal Me, $\phi_{\mathrm{Me}}$ ) of the cobalt and silver [1518] (Table 1) satisfy the condition (1), and consequently the occurrence of UPD phenomenon should not be rejected.

$$
\phi_{\mathrm{S}}>\phi_{\mathrm{Me}}
$$

In addition to this hypothesis, it could be predicted that if this phenomenon occurs, cobalt atoms would be adsorbed on the substrate surface as completely discharged species, because the difference of electronegativities between the substrate $\left(\chi_{\mathrm{Ag}}=1.86\right)$ and adsorbate $\left(\chi_{\mathrm{Co}}=1.80\right)$ meets the condition reported by Schultze and Koppitz [19], that is to say:

$$
\left|\chi_{\mathrm{Ag}}-\chi_{\mathrm{Co}}\right|<0.5
$$

It is important to emphasize that this hypothesis is supported by the results obtained with a similar system formed by cobalt on a gold substrate, even though this latter does not meet the condition of (1) using data of Trasatti [15]; however, Mendoza-Huizar et al. in 2002 [20], Flis-Kabulska in 2006 [21], and more recently Montes-Rojas et al. [22] have undertaken to prove the formation of said system.

It is important to consider that different authors [2325] have claimed that cobalt electrodeposition is thought to occur via the formation of $\mathrm{CoOH}^{+}$and $\mathrm{Co}(\mathrm{OH})_{2}$ species. The hydroxide species formation depends on the $\mathrm{pH}$ of the deposition bath. On the one hand, for solution $\mathrm{pH}$ lower than 4.1, the authors proposed that $\mathrm{Co}^{2+}$ and $\mathrm{OH}^{-}$react producing $\mathrm{CoOH}^{+}$"unstable complex." It is followed by the reduction of this complex and its reaction with adsorbed hydrogen to form metallic Co. On the other hand, for solution $\mathrm{pH}$ between 4 and 4.5 , the authors proposed that $\mathrm{Co}^{2+}$ and $\mathrm{OH}^{-}$react producing cobalt hydroxide $\left(\mathrm{Co}(\mathrm{OH})_{2}\right)$. This compound is reduced to produce metallic Co. More recently,
Pradhan et al. [24] and Matsushima et al. [25] have detected increased proportion of cobalt hydroxide species $\mathrm{CoOH}^{+}$and $\mathrm{Co}(\mathrm{OH})_{2}$ that are adsorbed on a platinum substrate before giving rise to cobalt deposit formation when using solution of $\mathrm{pH}$ 4.1. However, these results have not been checked with solutions with higher $\mathrm{pH}$.

One of the tools used in this type of works is quartz crystal microbalance coupled with electrochemical techniques (Electrochemical Quartz Crystal Microbalance, EQCM), because this technique allows detecting very small mass variations produced during the UPD process [26-28] or in the first stages of the growth of one metal, thanks to the piezoelectric effect of a thin quartz sheet covered on both sides by a thin metal film used as electrode.

This technique is very important for this kind of works according to Sauerbrey expression:

$$
\Delta f=-\left(\frac{f_{o}^{2}}{N \rho_{q}}\right) \Delta m,
$$

where the resonance frequency $\left(f_{o}\right)$ of a acoustic wave spreading in the sheet changes $(\Delta f)$ as a result of the mass variation on it $(\Delta m)$ produced during an electrochemical experiment. Variables in parentheses are constants characteristic of the material ( $\rho_{q}$ is quartz density $2.648 \mathrm{~g} \mathrm{~cm}^{-3}, N$ is the constant of quartz frequency $1.67 \times 10^{5} \mathrm{~Hz} \mathrm{~cm}^{-1}$, and $f_{o}$ is the resonance frequency in the fundamental mode) that may be grouped in a single constant called sensitivity factor $C_{f}$ :

$$
C_{f}=\frac{f_{o}^{2}}{N \rho_{q}}
$$

It is noteworthy that in order to be able to use Sauerbrey expression, mass gain process on the electrode should fulfill certain considerations.

(a) Deposits should be homogeneously distributed on the surface of the electrode.

(b) Deposit films should be tightly linked to crystal, and it should be as rigid as the quartz.

(c) Changes of frequency upon film deposition should be below $2 \%$ of resonance frequency.

The results shown in this work were obtained by studying the first stages of cobalt deposit formation on a silver substrate using quartz crystal microbalance coupled with electrochemical techniques.

\section{Experimental}

2.1. Solutions. The supporting electrolyte used in this work was prepared with $\left(\mathrm{NH}_{4}\right)_{2} \mathrm{SO}_{4}$ in $1 \mathrm{M}$ concentration. Cobalt solutions were prepared by dissolving $\mathrm{Co}\left(\mathrm{NO}_{3}\right)_{2}$ salts in tridistilled water, so that the metal ion concentration in the supporting electrolyte solution was $10^{-3} \mathrm{M}$. All solutions were adjusted to $\mathrm{pH} 9.3$ using reagent grade $\mathrm{NaOH}$ to diminish interferences of hydrogen evolution and to have the predominant species $\left[\mathrm{Co}\left(\mathrm{NH}_{3}\right)_{6}\right]^{2+}$ [29]. In addition, prior 
to the realization of experiments the solution was purged with high-purity nitrogen to remove dissolved oxygen, and the same gas was used to maintain inert atmosphere during experiments.

2.2. Electrodes. Working electrodes (Seiko) of $0.9653 \mathrm{~cm}^{2}$ geometric area were gold films supported on polished quartz discs with titanium underlayer to improve gold adherence to quartz, vibrating in the fundamental mode at $9 \mathrm{MHz}$. These electrodes were modified potentiostatically at $-960 \mathrm{mV}$ with a thin layer of silver using a solution of cyanides [30]. The counter electrode was a platinum wire with a greater area than the working electrode. Potentials were measured against and are quoted versus the $\mathrm{Ag}|\mathrm{AgCl}| \mathrm{NaCl} 3 \mathrm{M}$ reference electrode. In addition, the sensitive factor in the Sauerbrey equation (4) was determined by chronoamperometric thallium deposition [29], and its value was very close to the theoretical value $\left(1.714 \pm 0.012 \times 10^{8} \mathrm{~Hz} \mathrm{~g}^{-1} \mathrm{~cm}^{2}\right)$. Finally, all experiments were performed at room temperature.

2.3. Apparatus. Electrogravimetric experiments were carried out using a PAR potentiostat-galvanostat model 273A coupled with a QCM EG and G-Seiko microbalance model QCA922.

\section{Results and Discussion}

3.1. Voltammetric Characterization of a Silver Substrate. Figure 1 shows a cyclic voltammogram $(j-E)$ obtained at an electrode of quartz crystal modified with $\mathrm{Ag}$ film in electrolytic solution. This voltammogram has some characteristics that indicate the absence of faradaic processes since the very small current observed between $-100 \mathrm{mV}$ and $-1000 \mathrm{mV}$ corresponds to charging of the electrical double layer, and this feature is a criterion for the cleanliness of the surface of our substrates. Only when the potential is scanned at values more negative than $-950 \mathrm{mV}$ a cathodic current associated with hydrogen evolution is observed.

\subsection{Voltammetric Characterization of UPD and OPD Zones.} With the purpose to identify the processes that take place in the UPD and OPD region of the Co/Ag system, a large potential region was scanned using metal solution. In Figure 2 a comparison is shown of the experimental voltammograms obtained at the silver electrode in both solutions, without (a) and with (b) Co(II) ions in solution.

The potential region, in Figure 2(b), was scanned between $-100 \mathrm{mV}$ and $-1000 \mathrm{mV}$. The potential scan started at $-100 \mathrm{mV}$ up to $-1000 \mathrm{mV}$, and then it was reversed to the initial value. During the direct scan it can be noted that the cathodic current only increases when the potential is more negative than $-980 \mathrm{mV}$, and this tendency still continues upon scan reversal. This current is associated with the process of cobalt deposit formation on the silver substrate because the potential region explored is more negative than the equilibrium potential $\left(E_{\mathrm{eq}}=-741 \mathrm{mV}\right)[20]$ which is known as overpotential region (OPD). In addition, in this same

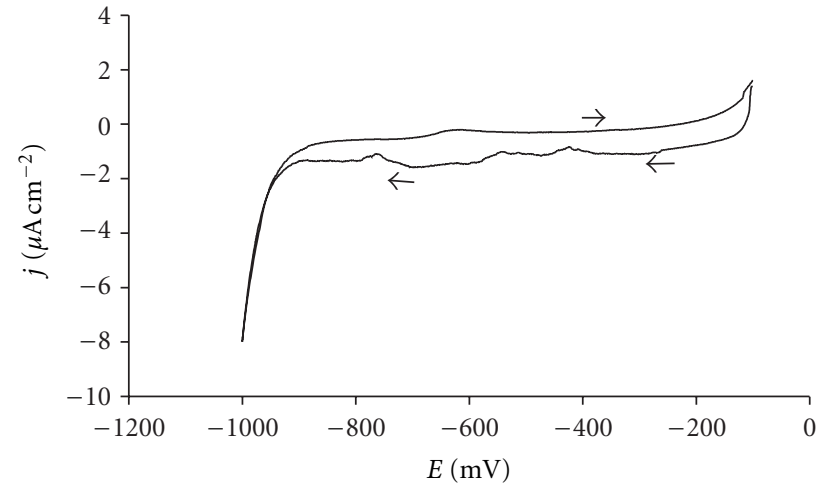

FIGURE 1: Voltammogram of $\mathrm{Ag} /\left[\left(\mathrm{NH}_{4}\right)_{2} \mathrm{SO}_{4}\right] 1 \mathrm{M}$ system at $\mathrm{pH} 9.3$ obtained at a scan rate of $5 \mathrm{mV} \mathrm{s}^{-1}$.

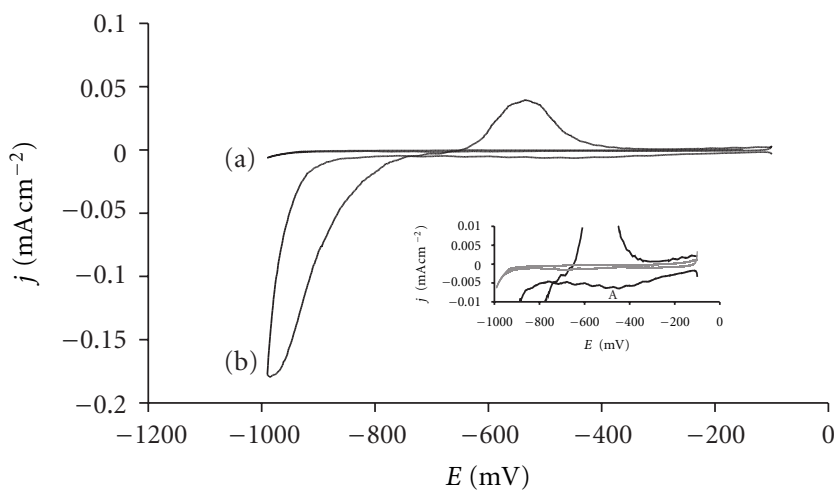

FIGURE 2: A comparison of two cyclic voltammetric curves obtained in the $\mathrm{Ag} / x \mathrm{M} \mathrm{Co}(\mathrm{II})+1 \mathrm{M}\left(\mathrm{NH}_{4}\right)_{2} \mathrm{SO}_{4}(\mathrm{pH}$ 9.3) system at two different Co(II) concentrations, (a) $x=0$ and (b) $x=1 \mathrm{mM}$. The scan potential rate was of $5 \mathrm{mV} \mathrm{s}^{-1}$, and the potential interval was $-100 \mathrm{mV}$ and $-990 \mathrm{mV}$. In the inset an amplification of the region comprising the peak of the alleged underpotential deposition is shown.

potential region the current measured must have a fraction associated with hydrogen evolution reaction.

In the reverse potential scan, one crossover of the cathodic and anodic branches at $-740 \mathrm{mV}$ is observed which is typical of the metal deposition process on a foreign substrate. According to different authors [20,31,32], in this crossover region it is possible to study nucleation processes, and the parameters of the nucleation-growth mechanism can be obtained. At potentials more positive than $-740 \mathrm{mV}$ one anodic peak appeared at $-548 \mathrm{mV}$ due to dissolution of the cobalt previously deposited during the direct potential scan, because if the negative scan limit is shortened, the intensity of this peak decreases. Finally, between $-400 \mathrm{mV}$ and $-100 \mathrm{mV}$ the current found is very small probably due to the fact that the substrate returned to its state before the experiments.

It is important to say that according to different authors [25] two current peaks were observed during cobalt dissolution from the electrode into the solution associated with dissolution of hydrogen rich cobalt phases that were previously formed during the cathodic sweep. In order to understand the nature of our peak, the curve $j-E$ was prepared from $\Delta f-E$ 


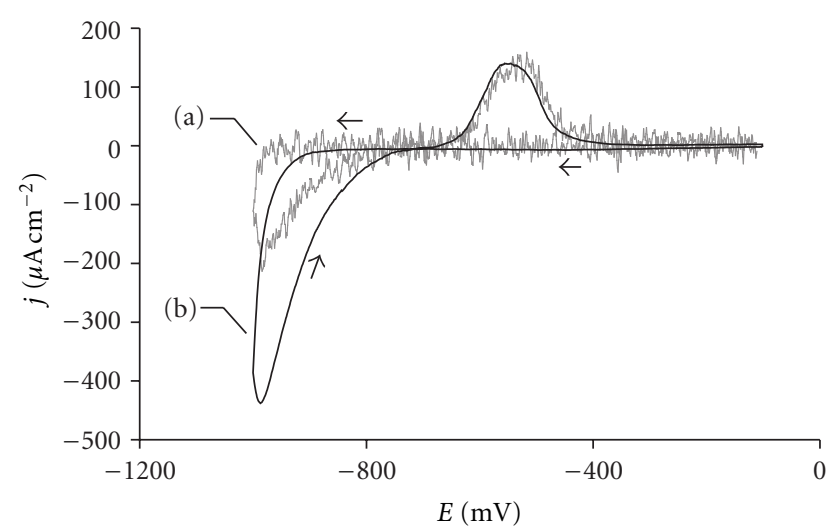

Figure 3: Comparison of $j-E$ curves obtained from $\Delta f$ - $E$ response (a) and directly from the experiment (b). The potential region scanned was $-100 \mathrm{mV}$ and $-1000 \mathrm{mV}$, and the scan potential rate was $5 \mathrm{mV} \mathrm{s}^{-1}$.

response and compared with the $j-E$ curve obtained directly from the experiments, Figure 3.

As may be seen the two curves are very well superimposed at potentials more positive than $-850 \mathrm{mV}$, when the scan is performed towards more negative potentials. However, at potentials more negative than $-850 \mathrm{mV}$ the curves are quite different. This difference remains even when the potential scan is reversed until the passage of the current to anodic quadrant. It is important to mention that the two curves are completely superimposed in the dissolution peak located at $-548 \mathrm{mV}$. This behavior is undoubtedly due to the fact that hydrogen formation reaction concurrent with cobalt reduction is greatly produced at potentials more negative than $-850 \mathrm{mV}$, but the formation of this species does not intervene in cobalt deposit dissolution, since the two curves are almost completely superimposed at peak $-548 \mathrm{mV}$. This proves that hydrogen-enriched cobalt phase is not formed, and that the deposit formed in these experiments is exclusively made up by Co species coming from soluble Co(II) species.

A more careful examination of the cathodic scan of curves in Figure 2 shows that there is a shoulder at $-483 \mathrm{mV}$ $\left(-4.9 \mu \mathrm{A} \mathrm{cm}^{-2}\right.$, peak $\mathrm{A}$ in the inset) probably associated with cobalt UPD process on the substrate given that this potential falls in the positive region with regard to $E_{\text {eq }}$. It is important to say that from the comparison between curves (a) and (b) it is clear that the peak $\mathrm{A}$ is indeed related to the system $\mathrm{Co} / \mathrm{Ag}$ and not to the electrolyte.

In order to determine the type of control limiting the peak at $-483 \mathrm{mV}$, different experiments were carried out at variable scan rates, and a logarithmic curve of absolute current values associated with peak $A$ as a function of scan rate was prepared as shown in Figure 4.

As can be seen, the absolute current value $(|j|)$ of peak A increased with the scan rate increase $(v)$, and the relationship found between both is linear with a slope of 0.5 , which implies that this process is controlled by mass transport [33]. It is important to mention that this behaviour was reported by Mendoza-Huizar et al. [20] with Co/Au UPD process in the presence of chlorides due to three different contributions: an

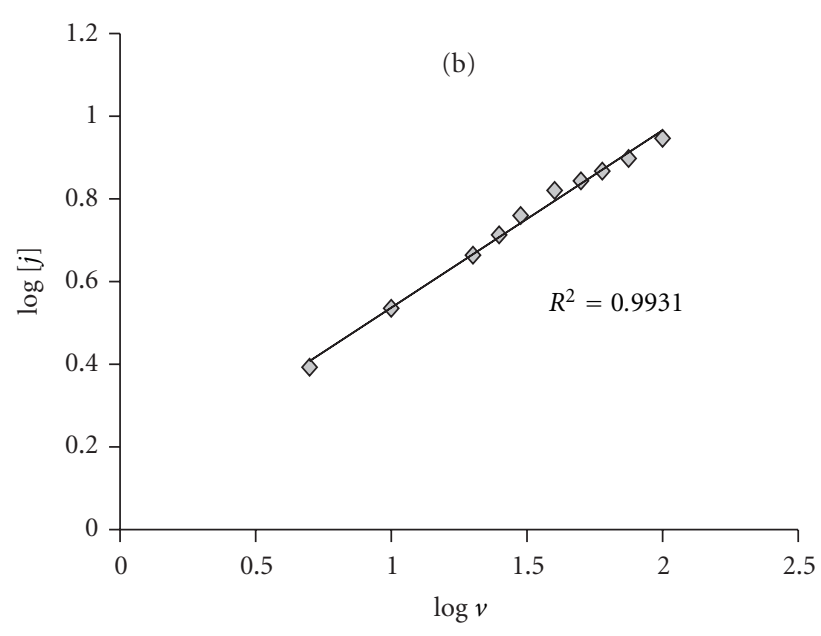

Figure 4: Logarithmic curve of absolute current peak $(j)$ as a function of scan rate $(v)$ for peak A.

adsorption process and 2D and 3D nucleation. However, in our case it seems that $\mathrm{NH}_{3}$ species plays the most important role in the deposition mechanism.

Additionally, Figure 5 shows the behaviour of the cathodic peak potential $\left(E_{\mathrm{pc}}\right)$ of peak $\mathrm{A}$ as a function of the scan rate within the range of $5-100 \mathrm{mV} \mathrm{s}^{-1}$. We can see that $E_{\mathrm{pc}}$ shifts towards more negative potentials with an increasing scan rate as a result of the system's irreversible behaviour [22].

\subsection{Electromicrogravimetric Characterization of UPD and} $O P D$ Regions. Figure 6 shows a typical frequency change curve $(\Delta f)$ as a function of the potential scan obtained concurrently with cyclic voltammogram. This response was obtained by scanning the potential between $-100 \mathrm{mV}$ and $-1000 \mathrm{mV}$.

According to this curve, during the negative scan from $-100 \mathrm{mV}$ to $-400 \mathrm{mV}$, there is no detectable change in frequency (see inset), so the current detected in the voltammogram (Figure 2) corresponds to the double-layer charging. However, a smooth variation in the frequency change is observed at potentials more negative than $-400 \mathrm{mV}$ but more positive than $-980 \mathrm{mV}$. We think that this behavior of the change of frequency in this potential region is associated with the process of formation of the Co deposit on the substrate. It is noteworthy that at this potential $(-980 \mathrm{mV})$ the change of frequency is around $-10 \mathrm{~Hz}$ (mass increases) which makes us think that this value may be related to the mass of an incomplete monolayer. On continuing the negative scan at potentials more negative than $-980 \mathrm{mV}$, a rapid decrease in frequency can be observed associated with the formation of massive deposit of Co on the monolayer. This process still continues when the scan reversal is produced at $-1000 \mathrm{mV}$ since the frequency keeps decreasing. Following scan reversal, the frequency remains constant between $-800 \mathrm{mV}$ and $-600 \mathrm{mV}$ until increasing (mass decreases) very rapidly in the region ca. -600 to $-500 \mathrm{mV}$ corresponding to anodic dissolution of Co deposit. Finally, the dissolution of Co traces 


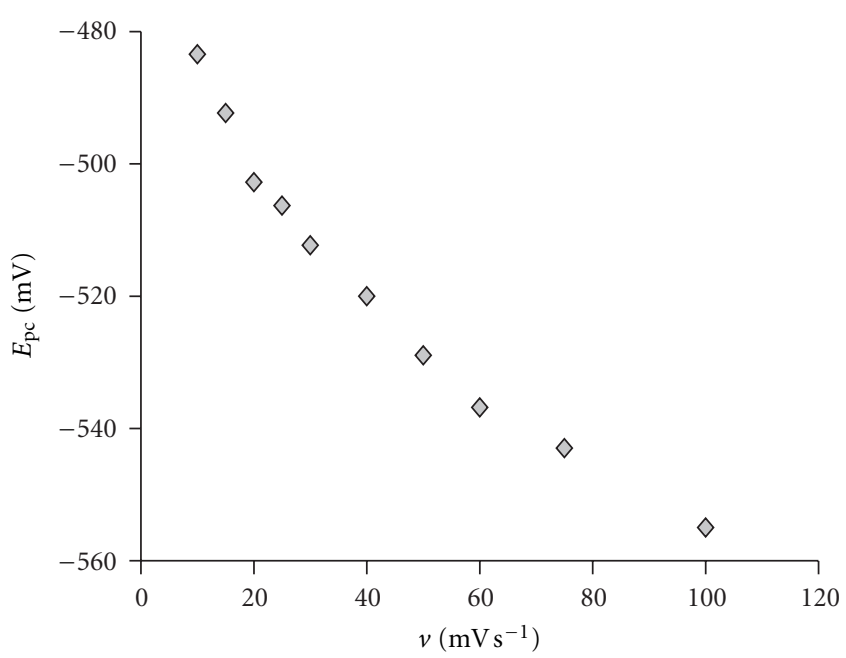

FIGURE 5: Current density curve of peak $(j)$ as a function of the scan rate $(v)$ for process $\mathrm{A}$; the data were obtained from experiments in the conditions as in Figure 2.

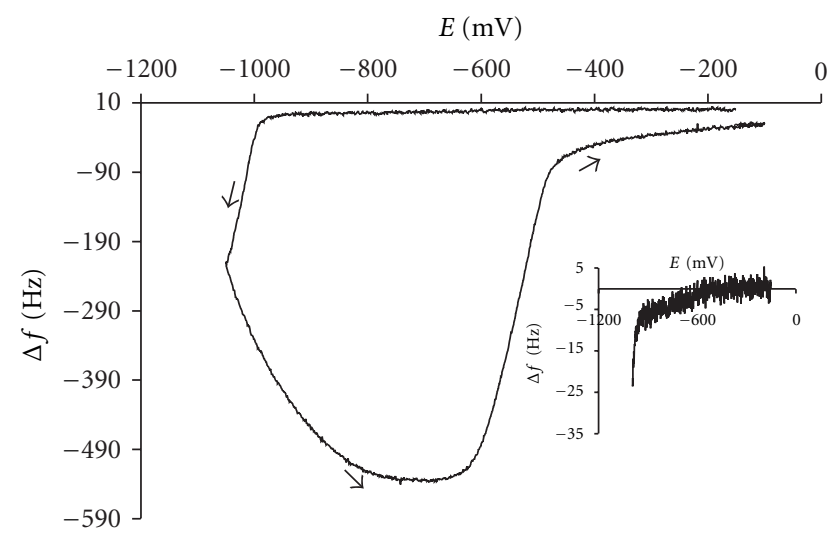

Figure 6: Frequency change $(\Delta f)$ curves as a function of the potential for $\mathrm{Ag} /\left[\mathrm{Co}^{2+}\right] 1 \mathrm{mM}$, $\left[\left(\mathrm{NH}_{4}\right)_{2} \mathrm{SO}_{4}\right] 1 \mathrm{M}$ system, and $\mathrm{pH} 9.3$ when scanning the potential until $-1050 \mathrm{mV}(-)$ and $-965 \mathrm{mV}(---)$ at $5 \mathrm{mV} \mathrm{s}^{-1}$.

on the substrate takes place partially since the frequency increases (mass decreases) slowly at potentials more positive than $-500 \mathrm{mV}$ until reaching almost the original value of $\Delta f$ in the double-layer region.

It should be emphasized that deposition processes of cobalt take place between $-500 \mathrm{mV}$ and $-980 \mathrm{mV}$; however, the peak $\mathrm{A}$ is not located in this potential region, consequently it seems that this peak is associated with another process.

Additional curves $\Delta f$ - $t$ were obtained at different negative potential steps $\left(E_{2}\right)$ which are shown in Figure 7.

These curves $\Delta f$ - $t$ show two behaviors in accordance with potential regions.

(a) $E_{2}$ is more positive than $-500 \mathrm{mV}$. These curves show no variations of $\Delta f$ which implies that there is no variation of mass on the substrate. According to these results, peak $\mathrm{A}$ is not necessarily associated with the cobalt deposition process, because it is not accompanied by an important change of frequency (mass). Based on these results, it is possible to establish that the interaction between Co and Ag is not as important as to give rise to the UPD process, and thereby they can be excluded safely.

(b) $E_{2}$ is more negative than $-500 \mathrm{mV}$. The $\Delta f$ changes slowly until $\Delta f$ attains a plateau that depends on the cathodic limit potential (see Figures 7(a) and $7(\mathrm{~b})$ ), and this plateau is only observed when the cathodic potential is more negative than $-900 \mathrm{mV}$ but more positive than $-980 \mathrm{mV}$ (see Figure $7(\mathrm{c})$ ). This behavior of $\Delta f$ implies that the Co submonolayer formed may give rise to even more compact structure as has been mentioned for $\mathrm{Pb} / \mathrm{Au}$ [34], for which reason $\Delta f_{\max }$ values obtained by voltammetry and chronoamperometry do not match. In addition, we assume that this plateau is associated with the total formation of a Co monolayer on the Ag substrate since the $\Delta f$ value is approximately $22 \mathrm{~Hz}$ (see Figure 8).

So the potential region in which the Co monolayer is formed on the substrate is limited by potentials more negative than $-500 \mathrm{mV}$ but more positive than $-980 \mathrm{mV}$. According to these results, the first stages of cobalt deposit formation on the silver substrate seem not to follow an underpotential process, but rather the behavior of the $\mathrm{Co} / \mathrm{Au}(111)$ system, reported by Kleinert et al. [35], who established that the initial stages of Co deposition take place by gradually decreasing the potential from $-0.45 \mathrm{~V}$ to $-0.8 \mathrm{~V}$ to overcome the high overpotential but avoid fast growth.

3.4. Effect of Ammonia and Sulfate Ion Adsorption on Silver Substrate. It has been mentioned in the literature $[36,37]$ that the process of sulfate or ammonia ion adsorption on a substrate may take place in the underpotential region. With the aim of obtaining more information, the adsorption of these species was studied using only the solution of supporting electrolyte.

Concomitantly with the voltammogram of Figure 1 , the response of frequency was obtained in the absence of metal ion in solution, Figure 9.

According to this curve (Figure 9(a)), if the potential scan is made toward potentials more negative than $-750 \mathrm{mV}$, an important decrease in frequency (increase of mass) is observed, which can be interpreted by the fact that supporting electrolyte $\left(\mathrm{NH}_{3}, \mathrm{NH}_{4}{ }^{+}\right.$, and $\mathrm{SO}_{4}{ }^{-2}$ species $)$ has a contribution in mass gain on the resonator, even without the presence of metal ion in the solution. However, if the same type of experiments is performed without adjusting the $\mathrm{pH}(\mathrm{pH}=$ 6.5) of the solution, that is, if there are only $\mathrm{NH}_{4}{ }^{+}$and $\mathrm{SO}_{4}{ }^{-2}$ ions, the response in frequency changes remarkably as can be seen in Figure 9(b).

In this experiment, frequency variation of the unadjusted solution (in the absence of $\mathrm{NH}_{3}$, curve b) passes the quadrant of positive values (mass decrease), implying the loss of mass on the silver electrode probably due to the desorption of sulfate anions. According to this, frequency evolution toward 


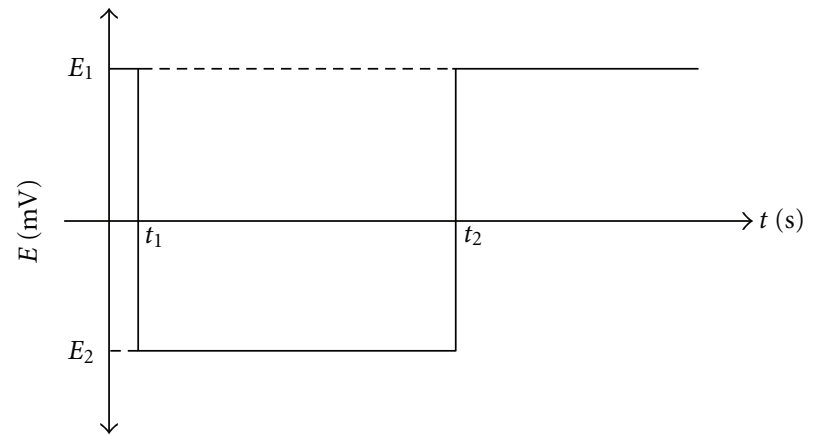

(a)

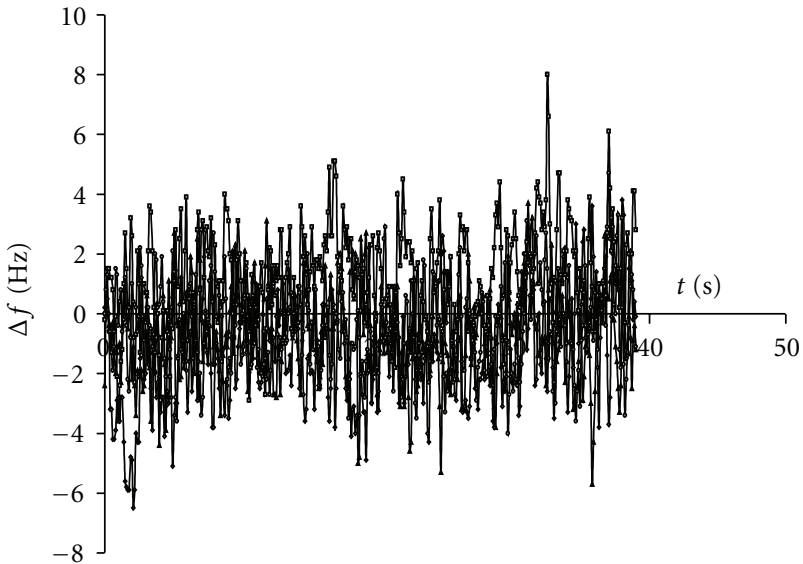

(b)

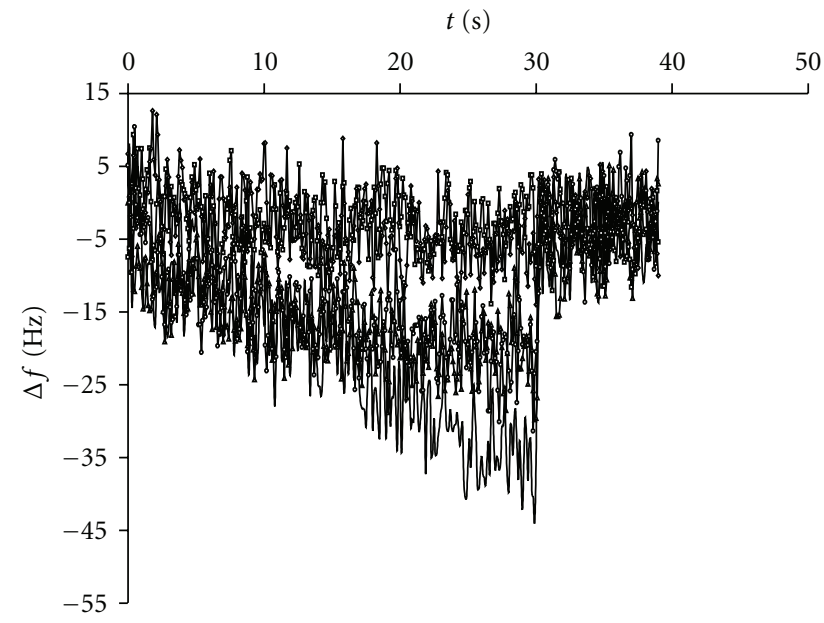

(c)

FIGURE 7: Responses of frequency change $(\Delta f)$ as a function of time for (a) peak A region (see Figure 2) and (b) for potentials more negative than peak A potential. Bath solution was made up of $\left[\mathrm{Co}^{2+}\right] 1 \mathrm{mM},\left[\left(\mathrm{NH}_{4}\right)_{2} \mathrm{SO}_{4}\right] 1 \mathrm{M}$, and $\mathrm{pH} 9.3$.

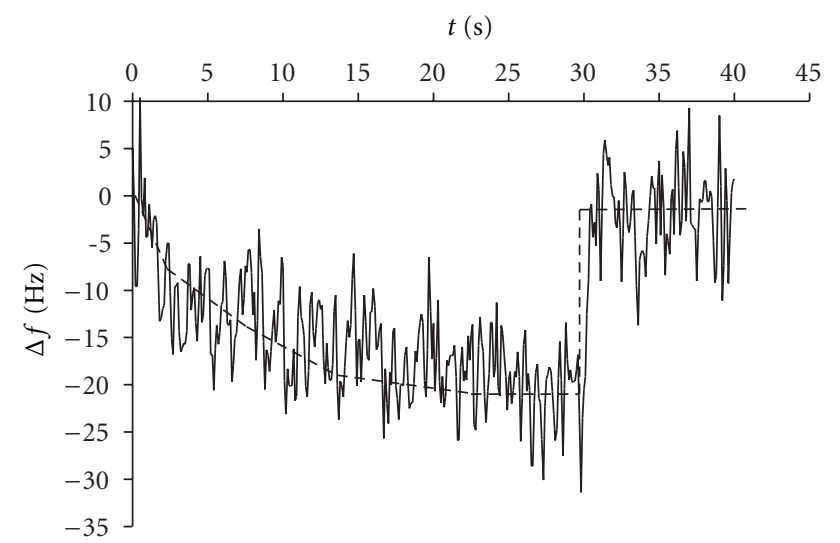

FIGURE 8: Monitored (-) and average (---) evolution of frequency change over time when potential pulses of $-980 \mathrm{mV}$ for cobalt deposition and $-150 \mathrm{mV}$ for oxidation are imposed for 30 and 10 seconds, respectively.

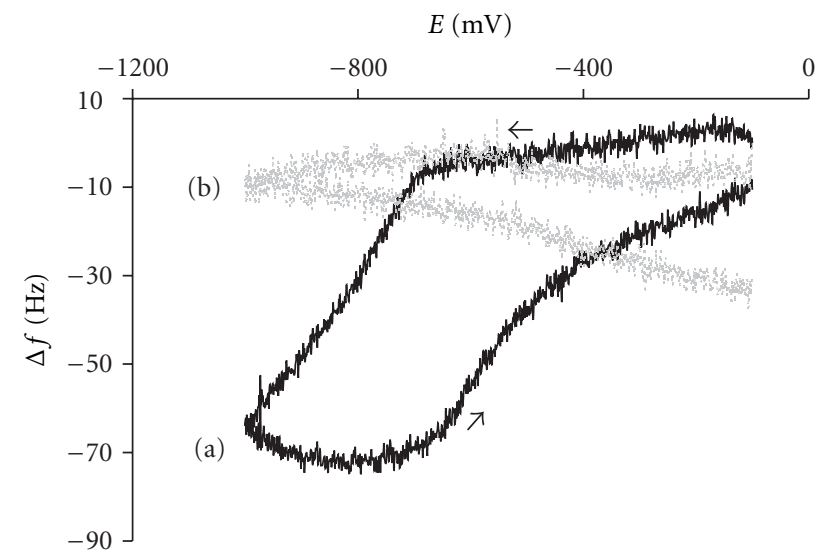

FIGURE 9: Frequency response as a function of potential scan for $\mathrm{Ag} /\left[\left(\mathrm{NH}_{4}\right)_{2} \mathrm{SO}_{4}\right] 1 \mathrm{M}$ system with (a) and without (b) $\mathrm{pH}$ adjustment obtained at a rate of $5 \mathrm{mV} \mathrm{s}^{-1}$. 


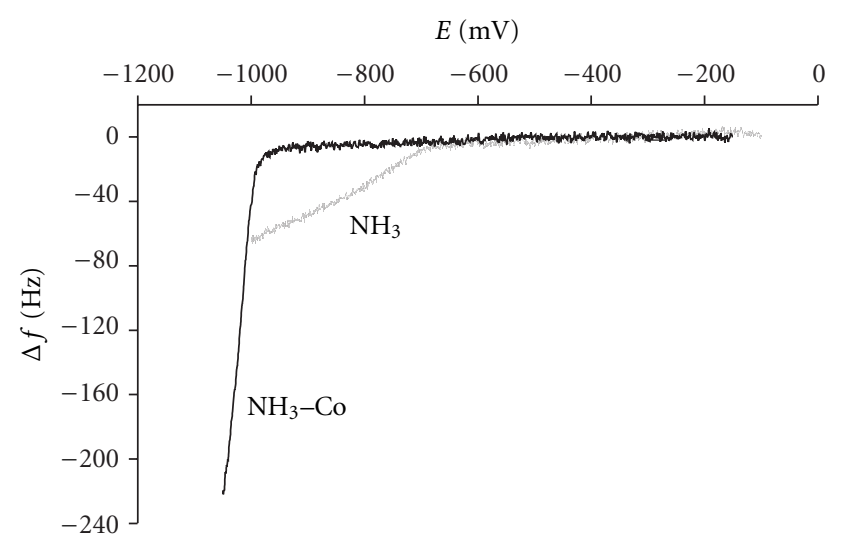

FIGURE 10: Fraction of the cyclic response of frequency change versus potential obtained at a rate of $5 \mathrm{mV} \mathrm{s}^{-1}$ using the following solutions: $\left[\left(\mathrm{NH}_{4}\right)_{2} \mathrm{SO}_{4}\right] 1 \mathrm{M}\left(\mathrm{NH}_{3}\right.$ curve $)$ and $\left[\mathrm{Co}^{2+}\right] 1 \mathrm{mM}+\left[\left(\mathrm{NH}_{4}\right)_{2} \mathrm{SO}_{4}\right] 1 \mathrm{M}\left(\mathrm{NH}_{3}-\mathrm{Co}\right.$ curve $)$ adjusted at $\mathrm{pH} 9.3$.

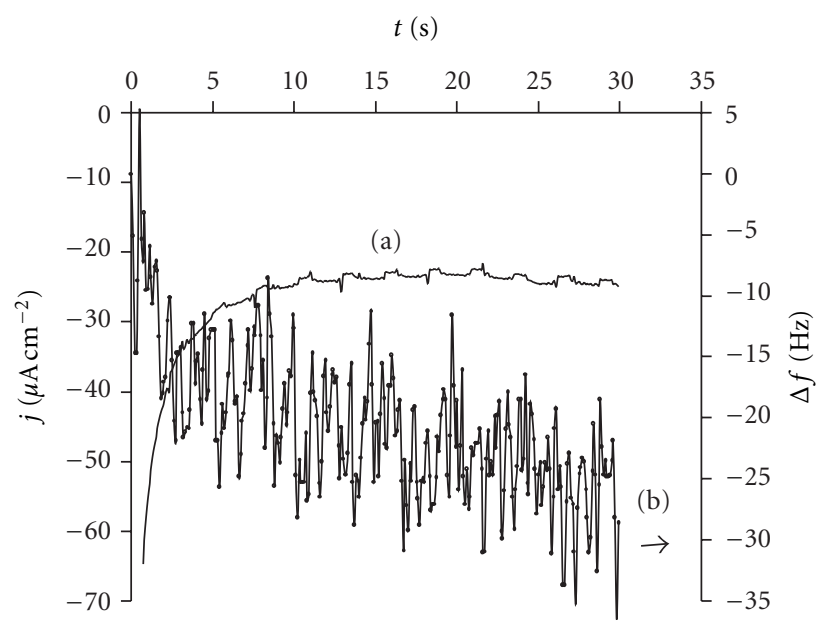

(A)

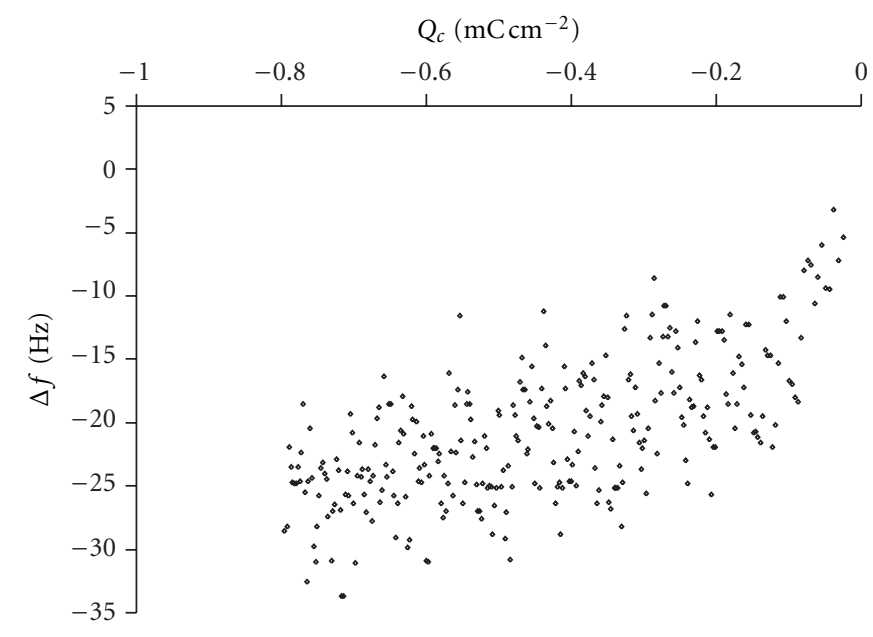

(B)

FIGURE 11: (A) Chronoamperogram (a) and profile of $\Delta f$ variation (b) over time, obtained by applying a potential pulse of -980 $\mathrm{mV}$ and using a solution formed by $\left[\mathrm{Co}^{2+}\right] 1 \mathrm{mM},\left[\left(\mathrm{NH}_{4}\right)_{2} \mathrm{SO}_{4}\right] 1 \mathrm{M}$, and $\mathrm{Ag}$ substrate as working electrode. (B) Frequency change diagram as a function of the charge, obtained from curves (a) and (b).

negative values, in the curve a, is due to the adsorption of ammonia molecules on the silver substrate. This is possible because of the zero charge potential $\left(E_{q=0}\right)$ of the silver substrate [38] $-1000 \mathrm{mV}$, so the adsorption of a species with a pair of free electrons, such as $\mathrm{NH}_{3}$ molecule, may take place on a positively charged surface, even though the potential is in the quadrant of negative values.

Now, in order to better define the contribution of ammonia in cobalt deposition, $\Delta f$ curves obtained in the absence and presence of metal ion were superimposed as a function of potential, Figure 10.

According to these curves, in the presence of metal ion ( $\mathrm{NH}_{3}$-Co curve) $\Delta f$ attributed to ammonia adsorption on silver substrate is observed to be practically suppressed, and instead, there is a very small variation of the order of ten $\mathrm{Hz}$ which increases as the potential approaches $-980 \mathrm{mV}$. This may be interpreted by the fact that ammonia adsorption on the silver substrate only takes place in the absence of metal ion at potentials more negative than $-750 \mathrm{mV}$. Another possibility implies that the process of ammonia adsorption takes place in a particular potential region, whereas in another region the adsorption of cobalt atoms occurs.

It has also been mentioned in the literature [23-25] that the process of cobalt deposit formation passes through formation of solid species precipitated on the electrode such as $\mathrm{Co}(\mathrm{OH})_{2}$; however, frequency variations detected in this work do not allow supporting this hypothesis. In addition, as will be seen further on, the intervention of $\mathrm{NH}_{3}$ species is more probable.

3.5. Determination of the Equivalent Molar Mass. These latter assumptions can be checked if apparent molar masses of the species adsorbed in different potential regions are determined, for which purpose curves from Figure 6 were used. 


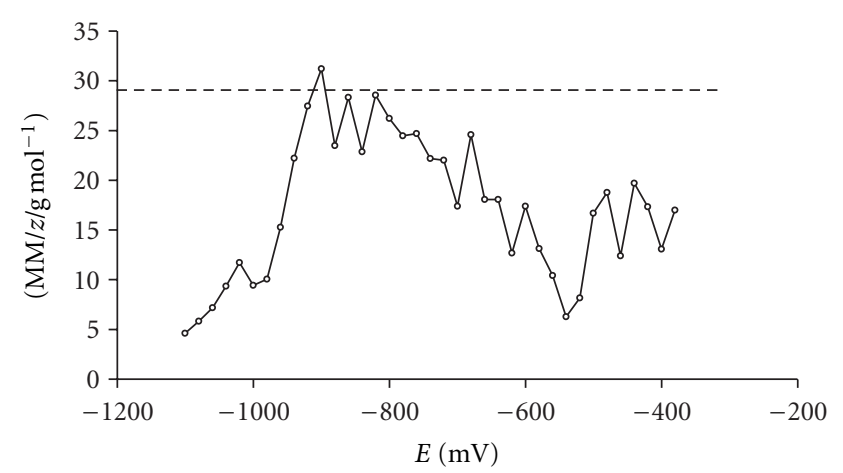

FIGURE 12: Equivalent molar mass curve as a function of the potential applied.

According to (5) resulting from the combination of Sauerbrey expression and second Faraday's law, $M M / z$ can be determined as

$$
\Delta f=\left(\frac{C_{f} \mathrm{MM}}{z F}\right) \Delta Q
$$

where $\mathrm{MM} / z$ is the equivalent molar mass of deposited species, and $\Delta Q$ is the charge density obtained using chronoamperometry, whereas the remaining terms keep their usual meaning.

So, frequency change curve was traced as a function of charge density, Figure 11, and from its slope $\mathrm{MM} / z$ was obtained by multiplying it with Faraday constant and sensitivity constant, as indicated by expression (5).

Equivalent molar mass $(\mathrm{MM} / z)$ obtained was graphed for a wide potential range, Figure 12 .

This figure shows that the equivalent molar mass $(\mathrm{MM} / z)$ adopts values dependent upon the potential region studied. Thus, between $-300 \mathrm{mV}$ and $-600 \mathrm{mV}$, for instance, the equivalent molar mass varies between 12 and $20 \mathrm{~g} \mathrm{~mol}^{-1}$; whereas between $-600 \mathrm{mV}$ and approximately $-950 \mathrm{mV}$, $\mathrm{MM} / z$ varies between 25 and $32 \mathrm{~g} \mathrm{~mol}^{-1}$. Finally, at the potential more negative than $-950 \mathrm{mV}$, the $\mathrm{MM} / z$ values are close to $10 \mathrm{~g} \mathrm{~mol}^{-1}$.

This behavior of $\mathrm{MM} / z$ confirms the assumptions from the above paragraphs, since in the potential region studied adsorption processes involving different species take place. For example, at potentials more positive than $-600 \mathrm{mV}$, ammonia is the adsorbed species, because its equivalent molar mass is comprised between 12 and $20 \mathrm{~g} \mathrm{~mol}^{-1}$ $\left(\mathrm{MM} / z\left(\mathrm{NH}_{3}\right)=17 \mathrm{~g} \mathrm{~mol}^{-1}\right)$; whereas at potentials between -600 and $-950 \mathrm{mV}$, the adsorbed species must be cobalt because its equivalent molar mass is comprised between 25 and $32 \mathrm{~g} \mathrm{~mol}^{-1}$ if two electrons are transferred $(\mathrm{MM} / z(\mathrm{Co})$ $=29.4 \mathrm{~g} \mathrm{~mol}^{-1}$ ). It is noteworthy that according to the information generated by this curve, there is no possibility of a partial charge transfer by cobalt atoms to the substrate, because the equivalent molar mass, in such case, should be superior to $30 \mathrm{~g} \mathrm{~mol}^{-1}$. Additionally, it is important to mention that equivalent molar masses above $30 \mathrm{~g} \mathrm{~mol}^{-1}$ indicative of intermediary cobalt hydroxide species, such as $\mathrm{CoOH}^{+}\left(\mathrm{MM} / z=37.965 \mathrm{~g} \mathrm{~mol}^{-1}\right)$ or $\mathrm{Co}(\mathrm{OH})_{2}(\mathrm{MM} / z=$

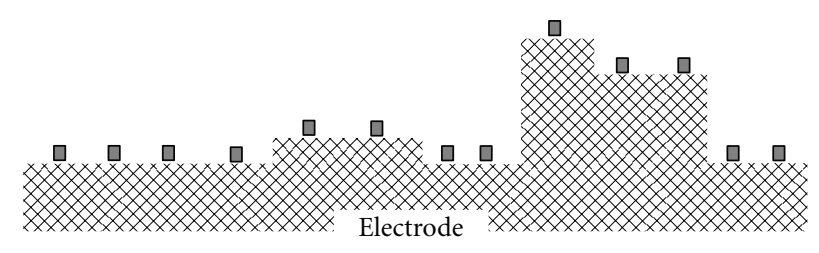

(a)

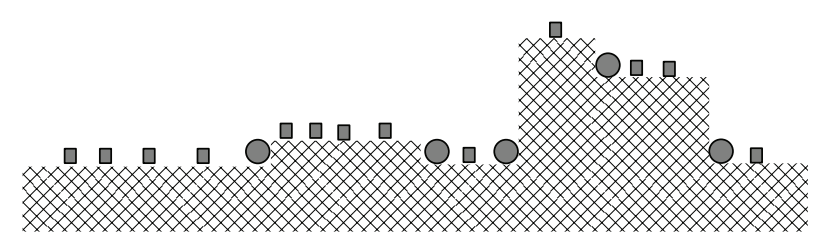

(b)

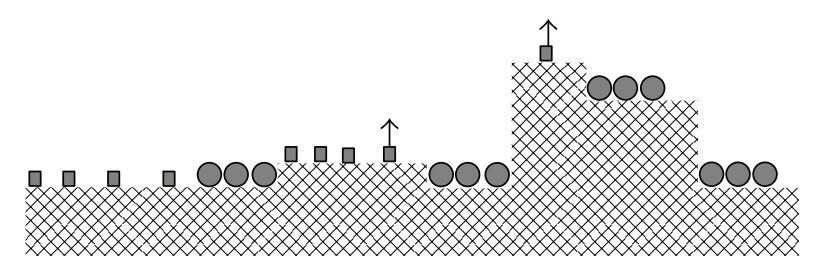

(c)

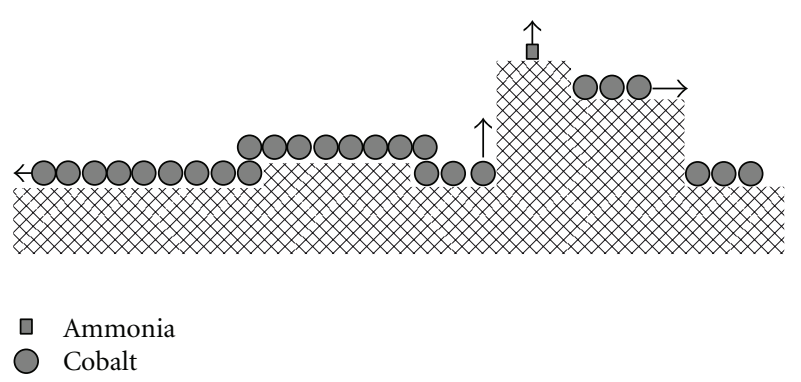

(d)

FIGURE 13: Schematic representation of cobalt underpotenial deposition process on a silver substrate, which takes into account ammonia adsorption: (a) adsorption of ammonia molecules at potentials more positive than $-600 \mathrm{mV}$; (b) and (c) processes of Co underpotenial deposit formation between -600 and $-920 \mathrm{mV}$; (d) process of total silver substrate covering by cobalt at potentials ranging between -920 and $-980 \mathrm{mV}$.

$46.465 \mathrm{~g} \mathrm{~mol}^{-1}$ ), were not obtained either, as mentioned by some authors for cobalt deposition in aqueous phase.

All this information is summed up in Figure 13 that outlines different stages of potential formation of cobalt deposit on the silver substrate.

According to this diagram, before the cobalt deposition process starts, (Figure 13(a)) ammonia is adsorbed on the silver substrate at potentials more positive than $-600 \mathrm{mV}$. When the cobalt deposition process initiates, at potentials more negative than $-600 \mathrm{mV}, \mathrm{NH}_{3}$ molecules are displaced from the silver substrate by metallic Co atoms (Figures 13(b) and $13(\mathrm{c}))$. Finally, silver substrate is completely covered by Co monolayer at potentials between -920 and $-980 \mathrm{mV}$ (Figure 13(d)). 


\section{Conclusions}

The initial stages of cobalt deposition process on a silver substrate were studied using cyclic voltammetry and chronoamperometry coupled with quartz microbalance in ammonia solution. The results obtained showed that during the Co deposit formation process on a silver substrate, no process of underpotential deposition is observed, even though the cobalt forms a monolayer on the substrate which starts from $-600 \mathrm{mV}$ to $-980 \mathrm{mV}$. During this process, the cobalt transfers its two electrons towards the electrode from soluble, and not solid species such as $\mathrm{Co}(\mathrm{OH})_{2}$. Furthermore, it was found that at potentials more positive than $-600 \mathrm{mV}$, ammonia adsorption takes place on the substrate's surface, and the above species leave its surface as this is occupied by cobalt.

\section{Acknowledgments}

This research was performed under the auspices of the Autonomous University of San Luis Potosí through Research Support Fund (C06-FAI-03-10.13) and of the Ministry of Public Education (SEP) through the Program for Teachers Improvement (PROMEP). A. L. D. Medrano thanks CONACyT for a scholarship.

\section{References}

[1] M. N. Baibich, J. M. Broto, A. Fert et al., "Giant magnetoresistance of (001)Fe/(001)Cr magnetic superlattices," Physical Review Letters, vol. 61, no. 21, pp. 2472-2475, 1988.

[2] A. E. Berkowitz, J. R. Mitchell, M. J. Carey et al., "Giant magnetoresistance in heterogeneous Cu-Co alloys," Physical Review Letters, vol. 68, no. 25, pp. 3745-3748, 1992.

[3] A. Gerber, A. Milner, I. Y. Korenblit, M. Karpovsky, A. Gladkikh, and A. Sulpice, "Temperature dependence of resistance and magnetoresistance of nanogranular Co-Ag films," Physical Review B, vol. 57, no. 21, pp. 13667-13673, 1998.

[4] J. Q. Xiao, J. S. Jiang, and C. L. Chien, "Giant magnetoresistance in nonmultilayer magnetic systems," Physical Review Letters, vol. 68, pp. 3749-3752, 1992.

[5] C. L. Chien, J. Q. Xiao, and J. S. Jiang, "Giant negative magnetoresistance in granular ferromagnetic systems (invited)," Journal of Applied Physics, vol. 73, pp. 5309-5314, 1993.

[6] L. Piraux, J. M. George, J. F. Despres et al., "Giant magnetoresistance in magnetic multilayered nanowires," Applied Physics Letters, vol. 65, no. 19, pp. 2484-2486, 1994.

[7] S. Kenane, E. Chainet, B. Nguyen, A. Kadri, N. Benbrahim, and J. Voiron, "Giant magnetoresistance in Co-Ag granular films prepared by electrodeposition," Electrochemistry Communications, vol. 4, no. 2, pp. 167-170, 2002.

[8] A. Canzian, G. Bozzolo, and H. O. Mosca, "Modeling of stable and metastable structures of $\mathrm{Co}, \mathrm{Cr}$, or Fe deposited on $\mathrm{Ag}(100)$ substrates," Thin Solid Films, vol. 519, no. 7, pp. 2201-2206, 2011.

[9] Y. J. Chen, W. Y. Cheung, I. H. Wilson et al., "Magnetic domain structures of $\mathrm{Co}_{22} \mathrm{Ag}_{78}$ granular films observed by magnetic force microscopy," Applied Physics Letters, vol. 72, no. 19, article 2472, 3 pages, 1998.

[10] Y. D. Zhang, J. I. Budnick, W. A. Hines, C. L. Chien, and J. Q. Xiao, "Effect of magnetic field on the superparamagnetic relaxation in granular Co-Ag samples," Applied Physics Letters, vol. 72, no. 16, pp. 2053-2055, 1998.

[11] J. A. De Toro, J. P. Andrés, J. A. González, J. P. Goff, A. J. Barbero, and J. M. Riveiro, "Improved giant magnetoresistance in nanogranular Co/Ag: the role of interparticle RKKY interactions," Physical Review B, vol. 70, no. 22, Article ID 224412, 6 pages, 2004.

[12] F. M. De Horne, L. Piraux, and S. Micote, "Fabrication and physical properties of $\mathrm{Pb} / \mathrm{Cu}$ multilayered superconducting nanowires," Applied Physics Letters, vol. 86, no. 15, Article ID 152510, 3 pages, 2005.

[13] D. M. Kolb, "Physical and electrochemical properties of metal monolayer on metallic substrates," in Advances in Electrochemistry and Electrochemical Engineering, H. Gerischer and C. W. Tobias, Eds., vol. 11, pp. 125-271, Wiley-Interscience Publication, New York, NY, USA, 1978.

[14] A. Aramata, "Underpotential deposition on single-crystal metals," in Modern Aspects of Electrochemistry, J. O. BocKris, R. E. White, and B. E. Conway, Eds., vol. 31, pp. 181-249, Plenum Press, New York, NY, USA, 1997.

[15] S. Trasatti, "Work function, electronegativity and electrochemical behaviour of metals. I. Selection of experimental values of work function," Chimica e Industria, vol. 53, pp. 559-564, 1971.

[16] R. Parsons, in Handbook of Electrochemical Constans, Butterworths, London, UK, 1959.

[17] H. B. Michaelson, "Work functions of the elements," Journal of Applied Physics, vol. 21, article 536, 5 pages, 1950.

[18] S. D. Ardage and E. Gileadi, in Electrosorption, Plenum Press, New York, NY, USA, 1967.

[19] J. W. Schultze and F. D. Koppitz, "Bond formation in electrosorbates-I correlation between the electrosorption valency and pauling's electronegativity for aqueous solutions," Electrochimica Acta, vol. 21, no. 5, pp. 327-336, 1976.

[20] L. H. Mendoza-Huizar, M. Palomar-Pardavé, and J. Robles, "Nucleation and growth of cobalt onto different substrates: part I. Underpotential deposition onto a gold electrode," Journal of Electroanalytical Chemistry, vol. 521, no. 1-2, pp. 95-106, 2002.

[21] I. Flis-Kabulska, "Electrodeposition of cobalt on gold during voltammetric cycling," Journal of Applied Electrochemistry, vol. 36, no. 2, pp. 131-137, 2006.

[22] A. Montes-Rojas, L. M. Torres-Rodríguez, and C. NietoDelgado, "Electromicrogravimetric study of underpotential deposition of Co on textured gold electrode in ammonia medium," New Journal of Chemistry, vol. 31, no. 10, pp. 17691776, 2007.

[23] S. P. Jiang and A. C. C. Tseung, "Reactive deposition of cobalt electrodes. III. Role of anions," Journal of the Electrochemical Society, vol. 137, no. 11, pp. 3387-3393, 1990.

[24] N. Pradhan, T. Subbaiah, S. C. Das, and U. N. Dash, "Effect of zinc on the electrocrystallization of cobalt," Journal of Applied Electrochemistry, vol. 27, no. 6, pp. 713-719, 1997.

[25] J. T. Matsushima, F. Trivinho-Strixino, and E. C. Pereira, "Investigation of cobalt deposition using the electrochemical quartz crystal microbalance," Electrochimica Acta, vol. 51, no. 10, pp. 1960-1966, 2006.

[26] O. Melroy, K. Kanazawa, J. G. Gordon, and D. Buttry, "Direct determination of the mass of an underpotentially deposited monolayer of lead on gold," Langmuir, vol. 2, no. 6, pp. 697-700, 1986.

[27] M. Hepel, S. Bruckenstein, and K. Kanige, "Expulsion of borate ions from the silver/solution interfacial region during 
underpotential deposition discharge of BiIII in borate buffer," Journal of the Chemical Society, Faraday Transactions, vol. 89, no. 2, pp. 251-254, 1993.

[28] B. K. Niece and A. A. Gewirth, "Potential-step chronocoulometric and quartz crystal microbalance investigation of underpotentially deposited $\mathrm{Tl}$ on $\mathrm{Au}(111)$ electrodes," The Journal of Physical Chemistry B, vol. 102, pp. 818-823, 1998.

[29] A. L. Donjuan-Medrano and A. Montes-Rojas, "Effect of the thickness of thallium deposits on the values of EQCM sensitivity constant," New Journal of Chemistry, vol. 32, no. 11, pp. 19351944, 2008.

[30] W. Blum and G. B. Hogaboom, in Galvanotecnia y Galvanoplastia: Dorado-Plateado-Niquelado-Cromado, CECSA, México, 1964.

[31] B. Soto, E. M. Arce, M. Palomar-Pardave, and I. Gonzalez, "Electrochemical nucleation of cobalt onto glassy carbon electrode from ammonium chloride solutions," Electrochimica Acta, vol. 41, pp. 2647-2655, 1996.

[32] S. Fletcher, C. S. Halliday, D. Gates, M. Westcott, T. Lwin, and G. Nelson, "The response of some nucleation/growth processes to triangular scans of potential," Journal of Electroanalytical Chemistry, vol. 159, no. 2, pp. 267-285, 1983.

[33] S. Srinivasan and E. Gileadi, "The potential-sweep method: a theoretical analysis," Electrochimica Acta, vol. 11, no. 3, pp. 321$335,1966$.

[34] A. Hamelin, "Lead adsorption on gold single crystal stepped surfaces," Journal of Electroanalytical Chemistry and Interfacial Electrochemistry, vol. 101, pp. 285-290, 1979.

[35] M. Kleinert, H. F. Waibel, G. E. Engelmann, H. Martin, and D. M. Kolb, "Dynamic EXAFS study of discharging nickel hydroxide electrode with non-integer Ni valency," Electrochimica Acta, vol. 46, no. 20-21, pp. 3119-3127, 2001.

[36] Z. Shi, S. Wu, and J. Lipkowski, "Coadsorption of metal atoms and anions: $\mathrm{Cu}$ upd in the presence of $\mathrm{SO}_{4}, \mathrm{Cl}^{-}$and $\mathrm{Br}^{-}$," Electrochimica Acta, vol. 40, pp. 9-15, 1995.

[37] O. R. Melroy, M. G. Samant, G. L. Borges et al., "In-plane structure of underpotentially deposited copper on gold(111) determined by surface EXAFS," Langmuir, vol. 4, no. 3, pp. 728732, 1988.

[38] G. Valette, "Double layer on silver single-crystal electrodes in contact with electrolytes having anions which present a slight specific adsorption: part I. The (110) face," Journal of Electroanalytical Chemistry and Interfacial Electrochemistry, vol. 122, pp. 285-297, 1981. 


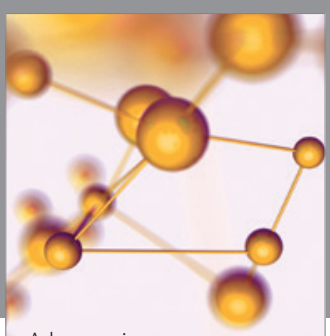

Physical Chemistry
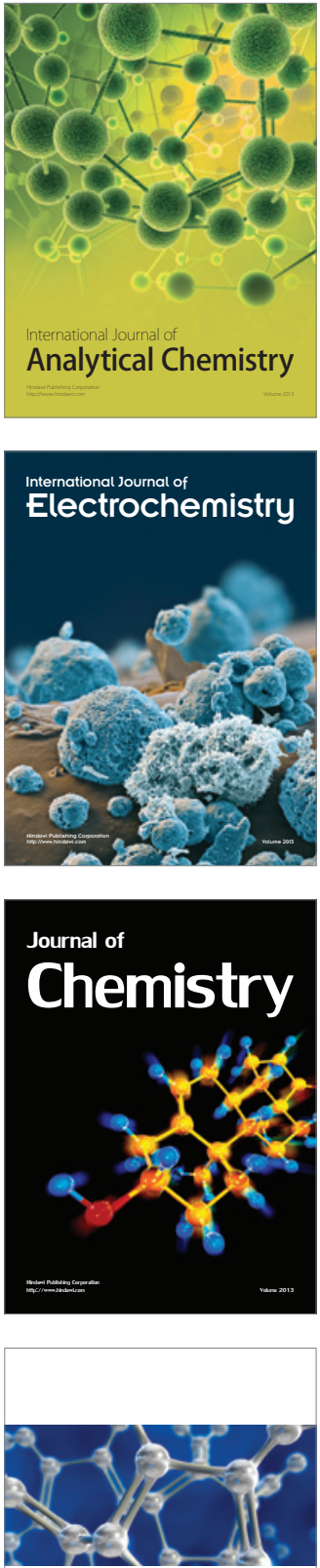

ISRN

Inorganic Chemistry

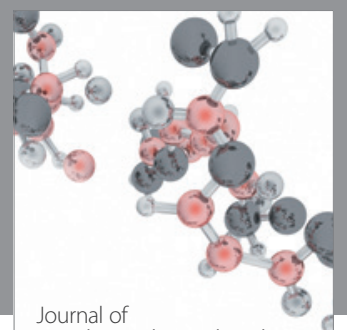

Analytical Methods in Chemistry

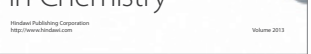

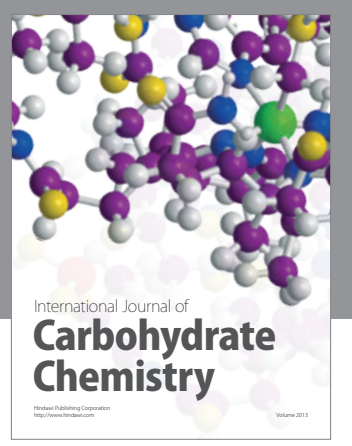
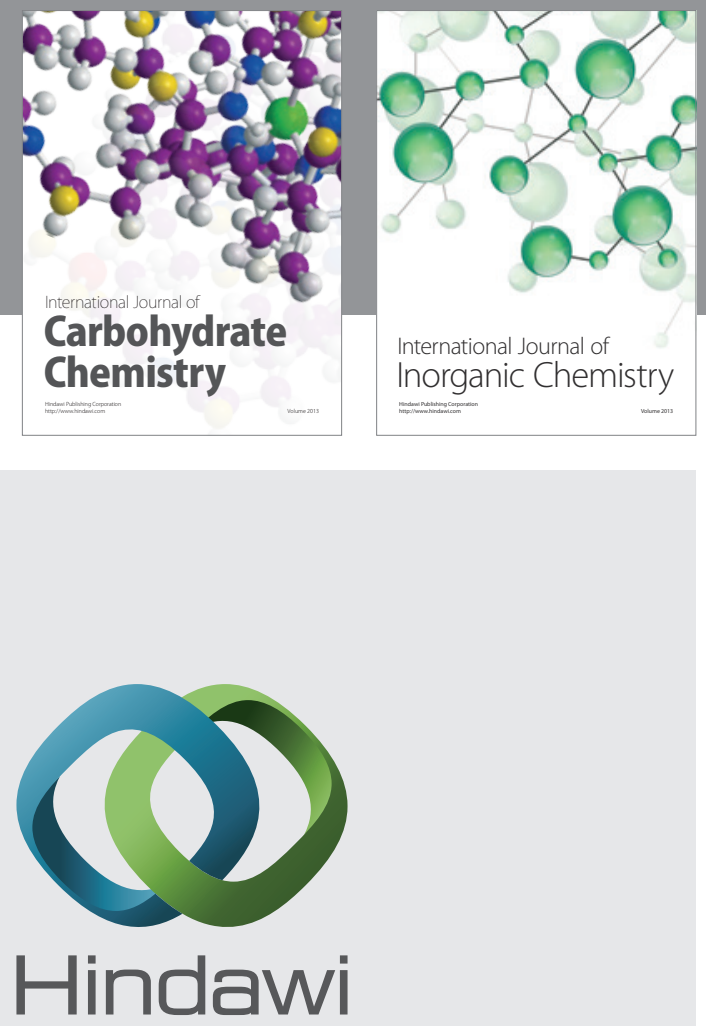

Submit your manuscripts at http://www.hindawi.com
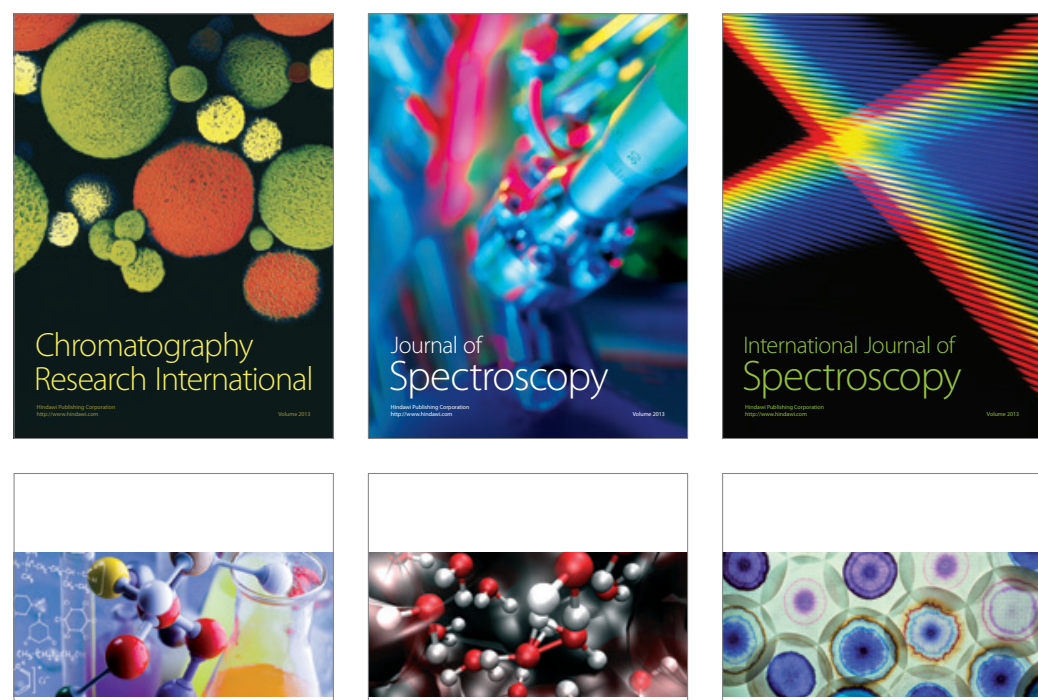

ISRN

ISRN

Organic Chemistry

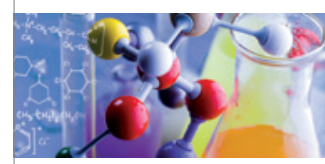

Physical Chemistry

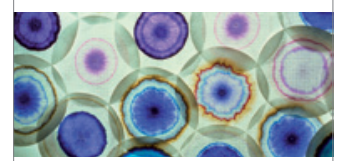

ISRN

Chromatography

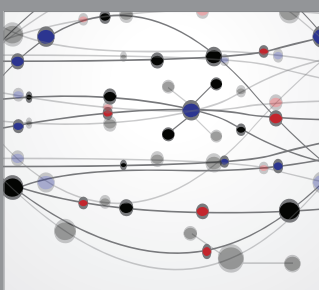

The Scientific World Journal
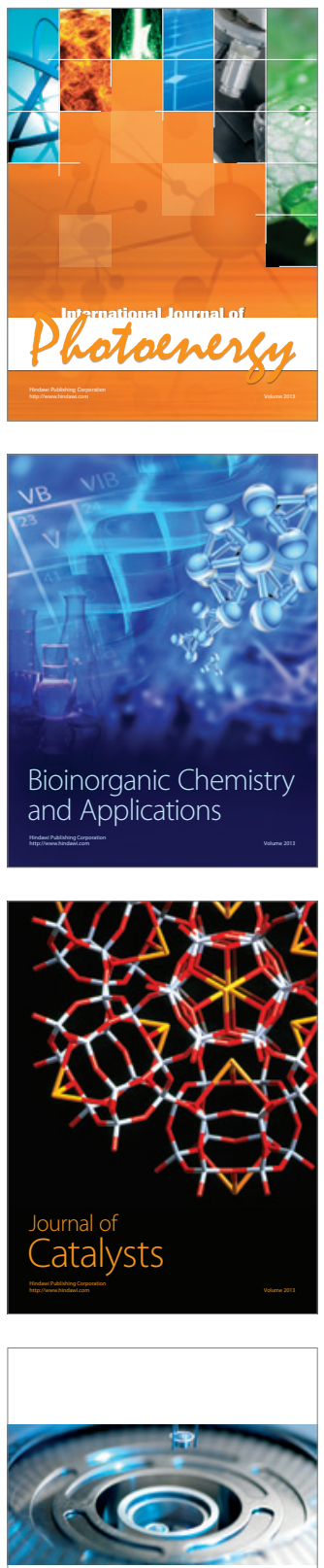

ISRN

Analytical

Chemistry 


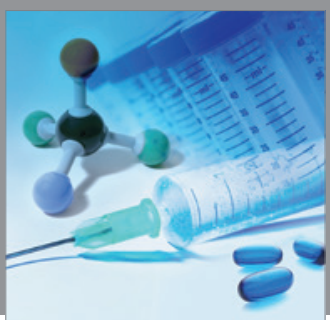

International Journal of

Medicinal Chemistry

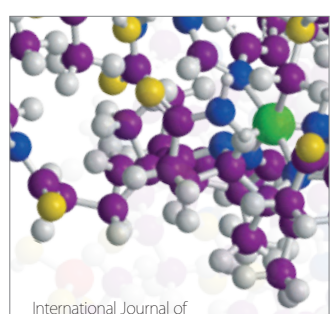

Carbohydrate Chemistry

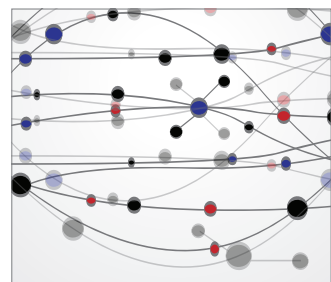

The Scientific World Journal
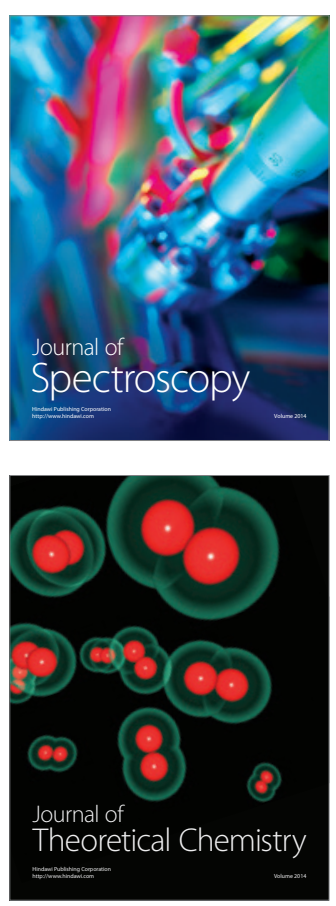
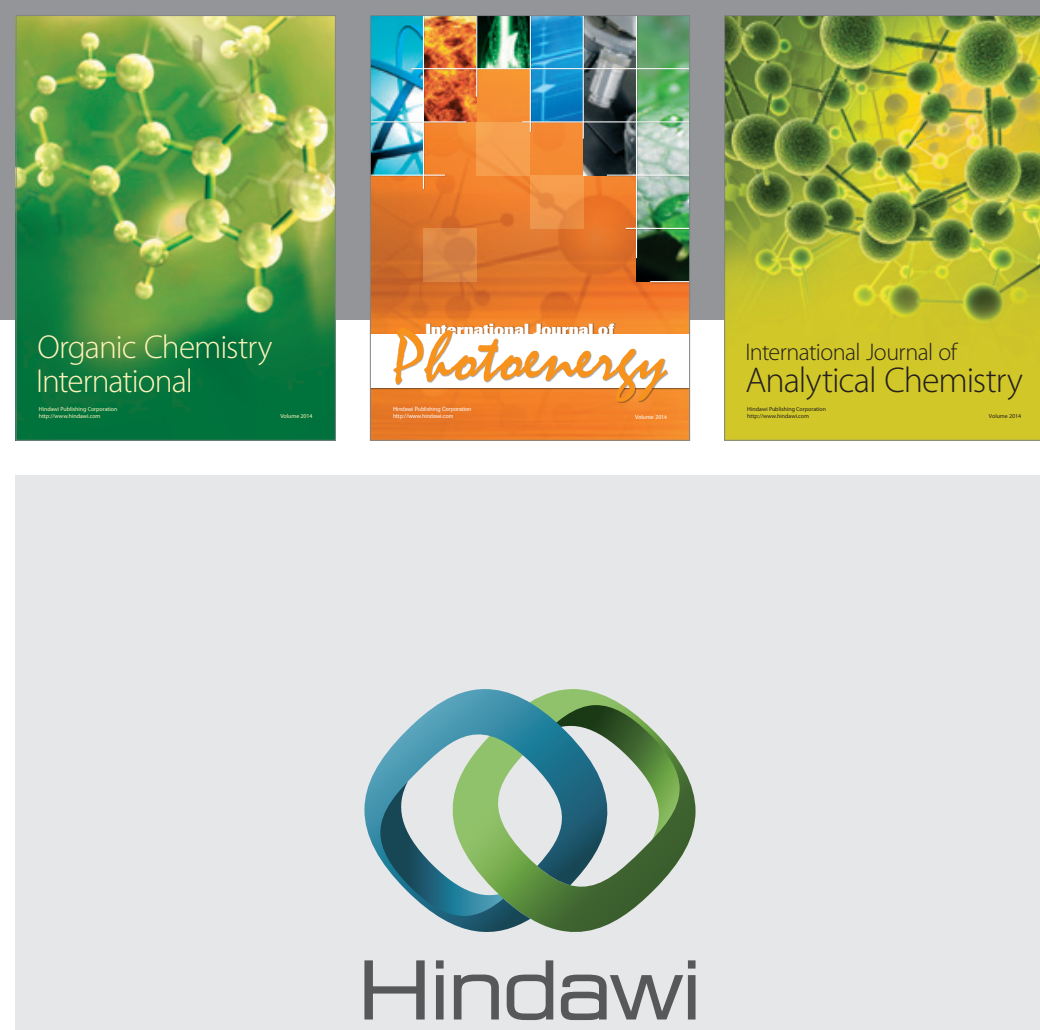

Submit your manuscripts at

http://www.hindawi.com
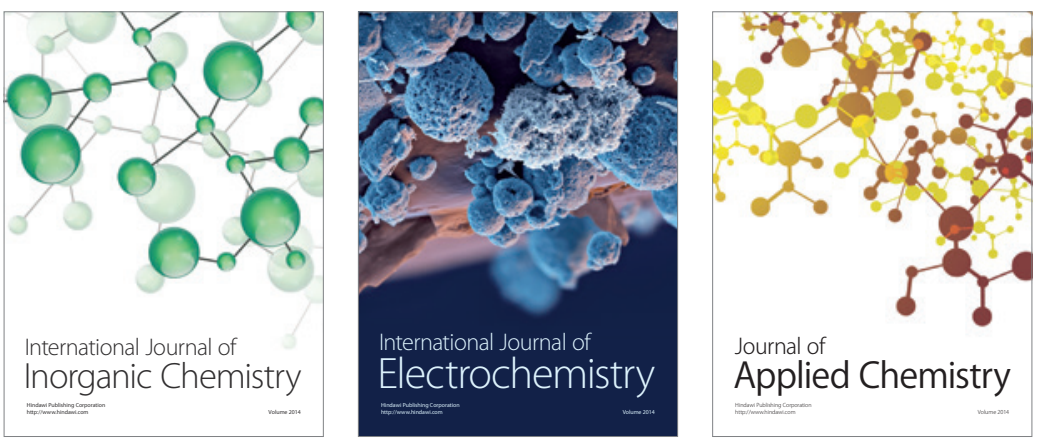

Journal of

Applied Chemistry
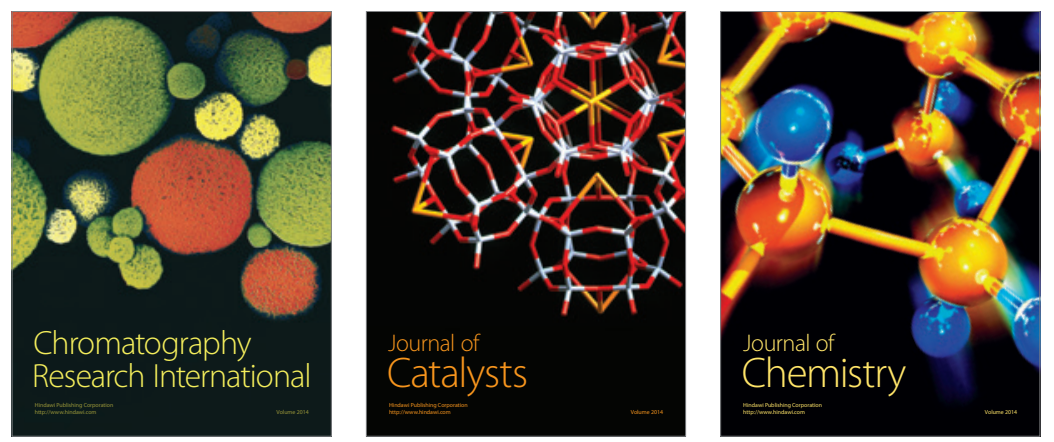
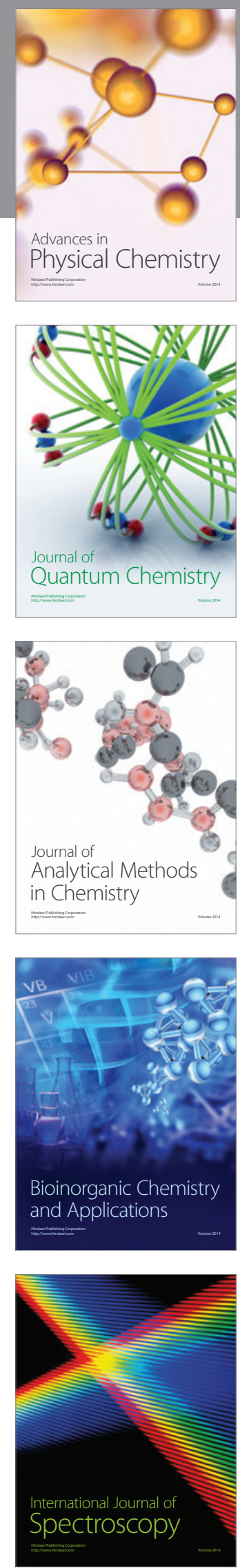